\title{
Extracellular vesicles as platforms for therapeutic microRNA delivery
}

Citation for published version (APA):

Cerqueira de Abreu, R. (2022). Extracellular vesicles as platforms for therapeutic microRNA delivery. [Doctoral Thesis, Maastricht University, Universidade de Coimbra]. Acco.

https://doi.org/10.26481/dis.20220221rc

Document status and date:

Published: 01/01/2022

DOI:

10.26481/dis.20220221rc

Document Version:

Publisher's PDF, also known as Version of record

\section{Please check the document version of this publication:}

- A submitted manuscript is the version of the article upon submission and before peer-review. There can be important differences between the submitted version and the official published version of record.

People interested in the research are advised to contact the author for the final version of the publication, or visit the DOI to the publisher's website.

- The final author version and the galley proof are versions of the publication after peer review.

- The final published version features the final layout of the paper including the volume, issue and page numbers.

Link to publication

\footnotetext{
General rights rights.

- You may freely distribute the URL identifying the publication in the public portal. please follow below link for the End User Agreement:

www.umlib.nl/taverne-license

Take down policy

If you believe that this document breaches copyright please contact us at:

repository@maastrichtuniversity.nl

providing details and we will investigate your claim.
}

Copyright and moral rights for the publications made accessible in the public portal are retained by the authors and/or other copyright owners and it is a condition of accessing publications that users recognise and abide by the legal requirements associated with these

- Users may download and print one copy of any publication from the public portal for the purpose of private study or research.

- You may not further distribute the material or use it for any profit-making activity or commercial gain

If the publication is distributed under the terms of Article $25 \mathrm{fa}$ of the Dutch Copyright Act, indicated by the "Taverne" license above, 
CHAPTER VI - Summary and General Discussion 
The therapeutic potential of extracellular vesicles (EVs) resides both in their innate regenerative potential or as a drug delivery system. Firstly, as a therapeutic agent, EVs have shown to be useful in several pathological contexts. Initially, attention was fuelled by the fact that EV cargo mimicked the content of the donor cell ${ }^{1}$ and therefore, the use of already established regeneration-inducing cell types, such as stem cells, sparked a paradigm shift towards EV use ${ }^{2-4}$. Stem cell transplantation for regenerative purposes has several shortcomings which the use of EVs eliminated or lessened ${ }^{5,6}$, such as the poor scalability of the systems, low engraftment/survival rates, oncogenic potential, elicitation of adverse immune responses, undefined mechanisms of action and even ethical concerns ${ }^{7}$. The discovery that EVs were key mediators of the therapeutic effects observed with stem cell therapies ${ }^{8-10}$ increased the interest in the field. The fact that EVs can bypass biological barriers ${ }^{11,12}$ render them particularly useful in the context of hard-to-reach tissues and organs, such as the heart or the brain. Furthermore, the low regenerative potential of these organs makes them a prime target for therapeutic EV usage. Finally, the possibility to engineer EVs can be harnessed to improve their bioactivity and/or targeting potential making them highly attractive therapeutic agents and/or drug delivery systems for already established therapeutic molecules ${ }^{13}$. In this thesis, two approaches were explored, in which we've made contributions to the field and wider scientific community. Firstly, in Chapter IV, we compared previously reported EV-enriching methodologies, with this study being the first to compare these approaches side-by-side for EV enrichment with miRNA. The functional effect of modifying EVs, both from a phenotypical outcome on recipient cells/tissues and an EV biology point of view was then explored. For the first time, we showed that while Exo-Fect is an efficient and reproducible method to load EVs with miRNA, it causes changes to EV biology which likely result in different cellular uptake and processing. This approach then inspired us to seek novel molecules capable of loading EVs with therapeutic molecules. Consequently, in Chapter V, a high-throughput quantitative screening methodology was developed and implemented for this purpose. Our results ultimately yielded two compounds which may be explored from a biotechnological perspective and constitute innovative contributions to the field. 


\section{Enhancement of EVs for therapeutic application}

In Chapter II, we performed an extensive literature review into the application of EVs in the cardiovascular setting. Given the epidemiological status of cardiovascular diseases (CVD $)^{14}$ and the lack of therapies capable of efficiently restoring cardiac function, EV-based therapies emerged as particularly promising alternatives. Despite the ongoing effort for the application of EVs to treat CVDs, their therapeutic efficacy remains limited ${ }^{15}$ mostly because in the damaged myocardium, EVs must contend with a harsh inflammatory environment ${ }^{16,17}$ and, regardless of the administration route, low accumulation rates ${ }^{18}$. Other limitations of EVbased technology pertain to the fact they are not easily trackable in vivo and although they may have some homing capacity for specific cell types, the majority of systemically administered EVs typically ends up in the liver and spleen ${ }^{18}$. Finally, the fact that the bulk of EVs that enter a recipient cell may end up being degraded by the endolysosomal system ${ }^{19}$, means that the effective cargo that may be released in the cytoplasm is variable and may be an order of magnitude lower than required to elicit a strong therapeutic effect. Nonetheless, recent studies have attempted to modify and enhance EV function(s) by engineering EVs in an attempt to overcome these obstacles ${ }^{20,21}$. In this Chapter, we identified four key areas where EVs may be enhanced to yield superior therapeutical outcomes: tracking, targeting, internalization and bioactivity.

To improve EV tracking, the EV membrane has been modified with fluorophores which allow for their tracking and monitorization in vivo, ultimately informing about the best dosage and administration regimens. However, a common issue with this type of approach is the fact that the tracking moieties may have longer half-lives than the EV itself and therefore caution is required when interpreting data pertaining to labelled EVs, as it may be prone to artefacts ${ }^{18}$. Other opportunities may arise in this field, however, as the development of triggerable systems, inspired, by synthetic nanoparticle approaches ${ }^{22}$, may allow for more specific visualization of EVs. Additionally, the development of fluorescence resonance energy transfer (FRET) in association with EVs may allow for specific tracking of the EVs in target tissues ${ }^{23}$.

A number of strategies has been employed to improve targeting and internalization of EVs into a given cell/tissue/organ. Several of these strategies explore the functionalization of the EV surface with peptides that aim to either bind specifically to certain cell types ${ }^{24,25}$, increasing 
EV availability in the tissue of interest, or facilitating endosomal escape 26,27 , increasing the cytoplasmatic availability of EV cargo in the recipient cell. Finally, enhancing EV bioactivity has been the area where most of the research has been conducted so far and several methods and approaches have been explored to that end ${ }^{28-32}$. Generally, the idea behind this type of EV enhancement entails loading EVs with therapeutically relevant molecules and harness their intrinsic capacity to cross biological membranes to effectively deliver the cargo in recipient cells. The dual use of EVs as drug delivery agents as well as therapeutic entities in their own right has become a new paradigm in EV research ${ }^{21,33}$. Most studies have focused on one of two possible approaches for the enrichment of EVs: modification of the donor cell followed by the isolation of EVs from the medium or post-isolation modification of EVs. While the former method tends to capitalize on the endogenous therapeutic potential of the donor cell ${ }^{34,35}$, the gamut of possible modifications is less controllable and/or limited given the technologies available for genetic manipulation of cells. Post-isolation methods are much more versatile given the fact that they often utilize EVs isolated from highly available sources such as urine or plasma or even milk and/or plants and the number of molecules used for the modification can be broader since no modification of the secreting cell is required However, the methods employed for modification may cause structural changes to $\mathrm{EVs}^{36,37}$, which may have unexpected effects in EV uptake and intracellular processing ${ }^{38}$. and ultimately their bioactivity. Which EV strategy is the most suitable to enhance cardiac function upon injury is still a matter of debate, and it was a question further explored in Chapters IV and V.

As previously established, EV action is contingent on effectively reaching the target cell. To this end, the interplay between EVs and endothelial cells is crucial, as these cells constitute the major interface between systemically delivered EVs and target tissues and organs. Consequently, microvascular health and development is key for effective EV action. On the other hand, as previously mentioned, non-coding RNAs are one of the bioactive cargoes present in EVs that can regulate the fate of the target cell. There is, therefore, an interest in understanding how all these entities interplay in health and disease. To this end, in Chapter III, we have explored the role of non-coding RNAs and EVs in microvasculature, particularly in the context of CVD. From this study, the role of non-coding RNA, especially microRNA, as highly relevant targets or treatments for cardiovascular repair and regeneration as been established. As discussed in Chapter II, several miRNAs have been used as therapeutic tools 
to regulate gene expression in various contexts. Presently, despite over 500 clinical trials being underway/concluded, involving the use of microRNA in humans, only a handful are being pursued as promising clinical tools ${ }^{39,40}$. So far, only a few clinical trials making use of miRNAs have entered phase $3 / 4$, and those that have intend to control endogenous levels of miRNA in order to produce a therapeutic effect. This is the case, for example, of a study using levosimendan to regulate the expression of miR-660-3p, miR-665 and miR-1285-3p, to ameliorate heart failure ${ }^{41}$. It remains to be seen whether the conjugation of the gene regulation power of microRNA can be translated into effective therapies using EVs as a delivery agent in clinic.

A caveat inherent to the compartmentalized view and study of EVs as presented in Chapters II and III is the fact that the EV is not construed as a whole entity. This is, EVs are mostly considered only in terms of their components (e.g. membrane, lumen, cargo) which may assessed or modulated. A more integrated conceptualization of EV study was alluded to in Chapter II, when the idea that multiple enhancements may be performed on the same vesicle was proposed. Nevertheless, even this multipronged approach lacks the context of the donor cell or the specific EV properties in question, for example. Perhaps a more holistic approach to EV study and application is necessary in order to achieve a true bionanoparticle with therapeutical value.

\section{Challenges in clinical application of EVs}

Having established the core concepts that demonstrate that EVs can be modulated to enhance their therapeutic activity, we explore in Chapter IV and Chapter V different methods and applications for EV enhancement. In Chapter IV, we showed that the commercial kit ExoFect is an efficient method to load EVs with a microRNA of interest and further showed that the modulated EVs were capable of downregulating the expression of target transcripts in vitro $^{38}$. In Chapter $\mathbf{V}$ we also showed that this technology is a valid tool for in vivo treatment of diabetic wounds, as it compares favourably with previous treatments regarding wound closure kinetics ${ }^{42}$. However, we also noted that the Exo-Fect reagent/protocol, despite its unknown composition, sticks to the EVs and elicits particle aggregation and cytotoxicity above the threshold of $0.5 \%(\mathrm{v} / \mathrm{v})$. These observations may explain why the uptake and processing 
of Exo-Fect-modulated EVs differs from their native ultimately contributing to changes in EV biology. Consequently, in Chapter V, we developed a novel screening assay to identify compounds capable of loading miRNA onto/into EVs. This high-throughput screening protocol yielded two compounds of undisclosed nature that appear to be highly efficient in loading EVs with pre-selected miRNAs. More work will need to be done to elucidate whether these molecules have the potential to reach Exo-Fect levels regarding their capacity to load the EVs with miRNA and whether they influence EV biology in the same way or not.

The desirable traits of an ideal EV transfecting agent are not yet well understood. From our results, it appears the cationic nature of the molecules may play a role in bringing the miRNA and EV membrane, both negatively charged. Importantly, while, theoretically, it would be more efficient to have a loading agent bring therapeutic molecules into the lumen of the EV, thereby conferring them protection, little is known of how to achieve that goal. Additionally, the technical difficulty of confirming a molecule was internalized into an EV is still a considerable obstacle in addressing this question. Moreover, effective concentrations of miRNA, or other therapeutic compounds of interest, are mostly based on studies that do not rely on EVs for delivery, hence adding another layer of complexity. In this regard, it has been found that EVs, from several sources, may carry endogenously less than 1 copy of a single miRNA per $\mathrm{EV}^{43}$ and that these EVs can yield beneficial phenotypical outcomes ${ }^{44}$ in regenerative situations. However, when loading EVs with exogenous material, most studies, including our own, can only ascertain an average of molecules per EV, which may not allow for accurate interpretation of data. For instance, it is possible that a few of the EVs in a loaded sample hold the majority of the therapeutic agent (low occupancy/high concentration model $\left.{ }^{43}\right)$. In this case, it is expected that the downstream functional studies resulting from using these EVs are more variable, depending on whether those specific enriched EVs were more internalized than their counterparts or not. Single-EV technologies and platforms currently in development will be key to answering these questions ${ }^{45}$. Also, as alluded to before, modifying EVs post-isolation may incur in unexpected biophysical alterations on affected EVs. For instance, our current work hypothesis regarding the study of Chapter IV indeed admits that Exo-Fect may stick to EV membranes, after transfection. Whether this can occur with other loading molecules, such as the compounds discovered in Chapter $\mathbf{V}$, is still not known. Interestingly, whether having loading agents stick to the EVs may appear strictly 
detrimental at first glance, by virtue of altering normal EV processing and biology, there may be serendipitous upsides to this process. In the case of Exo-Fect, a higher cellular uptake percentage and lower lysosomal colocalization point to the idea that while Exo-Fect may cover some portion of EV membrane, it via that mechanism that those EVs can interact with recipient cells in a way that leads to higher cytoplasmatic release of their contents. Finally, most of the studies herein discussed are centred around miRNA and similar nucleic acids. However, other therapeutically relevant drugs may be more effective, depending on the specific pathophysiological context of application. In those cases, despite some preliminary work ${ }^{46,47}$, it is still very much unknown whether the same principles as those current under development for small nucleic acids apply, or whether molecules of different natures, such as lipids or proteins, may require entirely distinct frameworks for EV loading, still remains to be explored.

From a technical standpoint, our work in Chapter IV sheds light on the difficulties of manipulating EVs and interpreting data. Several methodologies used for EV collection and modulation may introduce artefacts or otherwise alter normal EV biology. It is known that ultracentrifugation, the most used technique for EV purification, can collapse $\mathrm{EVs}^{48}$ and copurify unwanted contaminants ${ }^{49}$. Electroporation of EVs and microRNA causes precipitation of aggregates ${ }^{36}$. As we have also shown, Exo-Fect induces aggregation and quenching of fluorescent probes (both used for membrane and/or miRNA labelling) on EVs. The disparities caused by these and others techniques may confound EV research, particularly across different groups. These complications emphasize the need to improve and homogenize EVrelated practices, an effort that has been conducted by the International Society for Extracellular Vesicles (ISEV) ${ }^{50,51}$.

However, these are not the only obstacles hindering the translation of EV-based therapies into effective clinical alternatives. Another major bottleneck is still the scalability of EV production/collection systems. Many EV applications require the biosignature of their donor cell (i.e. the molecular, cytoplasmatic EV-sorted contents) to be therapeutic ${ }^{52,53}$, and as such the mass production of standardized EV batches needs to be established ${ }^{54}$. On the other hand, relying on the mass production of donor cells for EV harvesting is a costly and laborious process. Another alternative may be the utilization of primary human EVs. In Chapter $\mathbf{V}$ we 
also used human urine EVs, which may be one potential solution ${ }^{55}$. The use of these vesicles also allows for the use of EVs as autologous therapies reducing the risk of immunerejection/reaction by the recipient organism ${ }^{56}$. Other options have focused on the use of EVlike particles from plants ${ }^{57,58}$ or EVs from bovine milk ${ }^{59,60}$. These alternative sources of EVs may prove interesting and cost-efficient methods of obtaining high quantities of vesicles with a known bioactivity or prone to modification using bioengineering strategies.

Nevertheless, there is currently a race to the clinic focusing on EV-based platforms for drug delivery. In this regard, EVs compete with synthetic nanoparticles and viruses to some extent. Synthetic nanoparticles are generally more controllable systems, synthesized from known and reproducible reactions. Additionally, a large, easily modulable surface area makes them ideal candidates for compound conjugation ${ }^{61}$. However, typically the clearance of synthetic nanoparticles can either be too efficient, leading to poor biodistribution ${ }^{62}$, or too inefficient, leading to toxic accumulation in target tissues ${ }^{63}$. On the other hand, the efficiency of viralbased strategies for gene regulation is typically more efficient than EVs or nanoparticles. Nevertheless, potentially low loading capacity, off-target effects and immunogenicity concerns $^{64}$ still render some of these viral approaches unsuitable for clinic. As we have established previously, EVs have properties that distinguish them from other drug delivery platforms. Whether they can be harnessed effectively enough to bring EVs to the clinic in lieu of alternatives remains to be seen. 